\title{
ZERO DIVISORS AND COMMUTATIVITY OF RINGS
}

\section{J. E. MCLAUGHLIN AND ALEX ROSENBERG ${ }^{1}$}

1. Introduction. In this paper a study is made of the effect of the structure of the set of zero divisors on the associativity and commutativity of an alternative ring. A ring satisfies the alternative law if the associator $(x, y, z)=(x y) z-x(y z)$ is an alternating function of its arguments. In the case of a finite associative ring this problem has been studied by Herstein [2]. The theory of the radical of an alternative ring has been given by Smiley [12] and his definition will be used throughout.

By the center of an alternative ring $A$ we mean the set $C$ of all elements $c$ such that $(c, A, A)=(c, A)=0$. Here $(x, y)=x y-y x$ is the commutator of $x$ and $y$. If an alternative ring properly contains its center and all of its zero divisors lie in the center it will be shown that the set of zero divisors forms an ideal. Accordingly we first study alternative Zorn rings [8] where the set of left zero divisors forms a proper left ideal. Such a ring is a division ring modulo its radical and, conversely, in a Zorn ring which is a division ring modulo its radical the set of left zero divisors forms a left ideal. In $\$ 3$ we assume that all the zero divisors of an alternative ring lie in the center and then give sufficient conditions for the ring to be commutative and associative. In particular this turns out to be the case for an algebraic algebra over an algebraically closed or finite field. In the next section we assume only that the nilpotent elements lie in the center but here deal only with algebraic algebras. Under suitable hypotheses on the base field it is again true that the algebra is associative and commutative. In the last section we extend our results to real Banach algebras replacing the concept of zero divisor by that of topological zero divisor. In particular we show that a real Banach algebra with all its topological zero divisors in the center is the quaternions or commutative.

We wish to express our thanks to Professors I. Kaplansky and M. Ward for several conversations with regard to this paper.

2. Characterization of completely primary Zorn rings. We shall call a ring $A$ a Zorn ring if it is alternative and if every element $a$ is either nilpotent or has a left multiple $b a$ which is a nonzero idem-

Presented to the Society, April 25, 1952; received by the editors June 20, 1952.

1 This work was done while one of the authors (A. R.) was under contract with the U. S. Air Force. 
potent. ${ }^{2}$ An element $a$ of $A$ is called a left zero divisor if there exists a $d \neq 0$ in $A$ such that $a d=0$. Then we have the following

Theorem 1. Let $A$ be a Zorn ring in which the set $L$ of left zerodivisors forms a left ideal $\neq A$. Then $L=R$, the radical of $A$, and $A-R$ is a division ring.

REMARK. It is known from some recent work of Bruck and Kleinfeld that every alternative division ring is either associative or a Cayley-Dickson algebra. We should also like to point out that Theorem 1 generalizes a theorem of McCoy [9, Theorem 3].

Proof. Since $A$ is Zorn, $R$ is a nil ideal and so $R \subseteq L$. Now suppose $L$ is not nil, then by the Zorn assumption $L$ contains a nonzero idempotent $e$. Thus for all $a$ in $A, a=a e+(a-a e)$. The Theorem of Artin states that any subring of $A$ generated by two elements is associative. Therefore $(a-a e) e=0$, so $a-a e$ as well as $a e$ is in $L$. This implies that $A=L$, a contradiction. Therefore $L$ is nil and $L=R$.

By Lemma 18 of [8], $\bar{A}=A-R$ is also a Zorn ring. $\bar{A}$ contains no left zero divisors except 0 , for if $\overline{a d}=0$ in $\bar{A}$ then $a d$ lies in $R$. But then $(a d)^{n}=0$ for some minimal $n$. By the Theorem of Artin we can write $a\left[d(a d)^{n-1}\right]=0$, so either $a$ or $d$ is in $R$. Thus $\bar{A}$ is a Zorn ring with no left or right zero divisors except 0 , and therefore $\bar{A}$ is easily seen to be a division ring.

We next prove a partial converse of Theorem 1 .

Theorem 2. Let $A$ be a Zorn ring with radical $R$ and suppose that $A-R$ is a division ring. Then $L$, the set of left zero divisors in $A$, is either $R$ or all of $A$.

Proof. Suppose there is an $a$ not in $R$ such that $a d=0, d \neq 0$. We shall show that this implies $L=A$, and we begin by showing that all idempotents are in $L$.

Since $A-R$ is a division ring, $a$ is not nilpotent. Thus there is a nonzero idempotent $e=a^{\prime} a$ and we may assume $e a^{\prime}=a^{\prime}$. Using the Theorem of Artin we see that $f=a a^{\prime}$ is again a nonzero idempotent. Now if $e$ is not in $L, x=e x$ for all $x$ in $A$; in particular $d=e d$. Then we have $\left(a^{\prime}, a, d\right)=d$, and by (2.20) of [1], $\left(\left(a^{\prime}, a, d\right), a^{\prime}, a\right)=(e-f) d$. Thus $d=(e-f) d$. But $e-f=r$ is in $R$ so that $r^{n}=0$. A straightforward computation shows that $d=r^{m} d$ for all integers $m$, and therefore $d=0$, a contradiction. Hence $e$ is in $L$ and there is a nonzero element $g$ of $A$ such that $e g=0$. Now let $j$ be any idempotent. If $j$ does not lie in $L, x=j x$ for all $x$ in $A$. Therefore $g=j g=(j-e) g$ which again implies $g=0$. Thus all idempotents are in $L$.

\footnotetext{
2 For a discussion of Zorn rings see [7] and [8].
} 
Now let $x$ be any nonradical element of $A$. Again there are nonzero idempotents $e=x^{\prime} x$ and $f=x x^{\prime}$, and we have $x=x e+(x-x e)$. Since $x-x e$ lies in $R,(x-x e)^{k}=0$ for some minimal $k$. Suppose first $k>1$. Since $e$ and $f$ are in the subring generated by $x$ and $x^{\prime}$, the Theorem of Artin allows us to associate any expression involving only these four symbols. Thus we have $x(x-x e)^{k-1}=x e(x-x e)^{k-1}$. If $x$ is not in $L$, this yields $(x-x e)^{k-1}=e(x-x e)^{k-1}$. But $f(x-x e)=0$, and this gives $(x-x e)^{k-1}=(e-f)(x-x e)^{k-1}$ which as before implies $(x-x e)^{k-1}$ $=0$. Hence if $k>1, x$ is in $L$.

Now suppose $k=1$; then $x=x e$. There is a nonzero $d$ in $A$ such that $e d=0$, and therefore $(x, e, d)=x d$. But by (2.13) of [1]

$$
x(d x)=(x d) x=(x, e, d) x=(x, x e, d)=(x, x, d)=0 .
$$

So $x$ is in $L$ unless $d x=0$. But then $0=(d, x, e)=(x, e, d)=x d$, so that in this case also $x$ is in $L$. Thus every element of $A$ is in $L$.

It should be noted that we do not assume the presence of a unit. If, however, a unit is present the proof of Theorem 2 can be simplified considerably. ${ }^{3}$ Furthermore if a Zorn ring $A$ satisfies the hypothesis of Theorem 1, the right zero divisors are either $A$ or $R$. In the latter case $A$ has a unit. An example of a Zorn ring where the left zero divisors coincide with $A$ and the right zero divisors coincide with $R$ is furnished by the set of all $2 \times 2$ matrices with entries from a field and with their second column consisting entirely of zeros.

We now give examples to show that something like the Zorn assumption is needed to insure the validity of Theorems 1 and 2. Let $C$ be the ring of all polynomials with integer coefficients and zero constant term. Let $B$ be the direct sum of a countably infinite number of replicas of $C$. Finally let $A$ be the ring obtained by adjoining a unit to $B$ in the usual fashion $[10, \mathrm{p} .87]$. Then the zero divisors of $A$ form an ideal, namely the ring $B$, but $A$ is semi-simple.

The hypothesis of Theorem 2 is somewhat redundant. For if $A$ is an alternative ring with a nil radical $R$ and $A-R$ is a division ring, then $A$ is automatically a Zorn ring. However, if we merely assume that $R$ is nil and $A-R$ is a domain of integrity, Theorem 2 no longer holds. The following example is offered. Let $B$ and $C$ be the additive groups of order 2 and 3 respectively made into zero rings. Let $D$ $=B \oplus C$ and adjoin a unit to $D$ in the usual manner, using the ordinary integers. The resulting ring $A$ has $D$ for a radical and $A-D$ is the ring of integers. However the zero divisors in $A$ do not form an ideal.

${ }^{3}$ Cf. the proof of Theorem 5 . 
3. Zero divisors in the center. By the center $C$ of a Zorn ring $A$ we mean the set of all $c$ in $A$ for which $(c, A)=(c, A, A)=0$. Suppose that all the left zero divisors lie in $C$; it is then clear that all the right zero divisors lie in $C$. If $A$ properly contains $C$ we have the following

LEMMA 1. Let $A$ be an alternative ring with all the zero divisors in the center $C$. If $A$ properly contains $C$ the zero divisors form an ideal.

Proof. If $u$ is a zero divisor it is clear that any multiple of $u$ is also a zero divisor. If $A$ is noncommutative there is a pair of elements $x, y$ in $A$ with $(x, y)=x y-y x \neq 0$. If $u$ is any zero divisor of $A$, an easy computation shows that $u(x y-y x)=0$. Hence the difference of any two zero divisors is again a zero divisor. A similar argument works if $A$ is nonassociative.

For the remainder of this section we shall assume that $A$ is a Zorn ring properly containing its center $C$ and that all zero divisors lie in $C$. There are now two possibilities; if the only zero divisor is 0 it is easily seen that $A$ is a division ring. On the other hand if nonzero zero divisors are present, the proof of Lemma 1 shows that every commutator and associator is a zero divisor and so an application of Theorems 1 and 2 and Lemma 1 yields the following: The set of zero divisors coincides with the radical $R$ of $A, A-R$ is a commutative associative field $F$, and the only nonzero idempotent of $A$ is its unit. Furthermore, by the Zorn assumption it follows that every element of $A$ not in $R$ has an inverse. In particular, therefore, $C-R$ is a field $Z$.

Since every associator and commutator is in the center, (2.7) and (2.9) of [1] show that the mapping $x \rightarrow D_{y z} x=(x, y, z)$ is a derivation. Again using the fact that all associators are in the center we obtain the following formula:

$$
D_{y z}\left(c x^{m}\right)=m c x^{m-1} D_{y z} x=m c x^{m-1}(x, y, z) \text { for } c \text { in } C[y, z] .
$$

By using (2.6) of [1] and the Theorem of Artin we see that in certain special cases the mapping $x \rightarrow D_{y} x=(x, y)$ is a derivation; in fact we have

$$
D_{y}\left(c x^{m}\right)=m c x^{m-1} D_{y} x=m c x^{m-1}(x, y) \quad \text { for } c \text { in } C[y] .
$$

We now consider the structure of $F$ over $Z$. First suppose $F$ is of characteristic zero. Assume that $A$ is nonassociative and that $F$ is of transcendence degree less than 3 over $Z$. Then there are three elements $x, y, z$ in $A$ such that $(x, y, z) \neq 0$. These map into elements $\bar{x}, \bar{y}, \bar{z}$ of $F$ and we may always assume, by relabelling if necessary, that for some minimal $n$ 


$$
\bar{f}(\bar{x}, \bar{y}, \bar{z})=P_{n}(\bar{y}, \bar{z}) \bar{x}^{n}+\cdots+P_{0}(\bar{y}, \bar{z})=0
$$

where $P_{i}$ lies in $Z[y, z]$ and $P_{n} \not \equiv 0$. Lifting $\bar{f}$ to $A$ we obtain a relation

$$
f(x, y, z)=Q_{n}(y, z) x^{n}+\cdots+Q_{0}(y, z)=0
$$

where $Q_{i}$ is in $C[y, z]$ and $Q_{n} \neq 0$. But then

$D_{y z} f=\left[n Q_{n}(y, z) x^{n-1}+\cdots+Q_{0}(y, z)\right](x, y, z)=g(x, y, z)(x, y, z)=0$.

Since $(x, y, z) \neq 0$ this shows that $g(x, y, z)$ is in $R$. But then $\bar{g}(\bar{x}, \bar{y}, \bar{z})$ $=0$ in $F$, contradicting the minimality of $n$. In case $A$ is associative but noncommutative a similar argument will show that the transcendence degree of $F$ over $Z$ is at least 2 .

Suppose now that $F$ is of characteristic $p \neq 0$. Then $p=p e, e$ the unit of $A$, is in $R$ so that by Lemma $1, p(x, y, z)=p(x, y)=0$, for any $x, y, z$ in $A$. Formulae (3.1) and (3.2) then show that $x^{p}$ is in $C$ for all $x$ of $A$. Thus $F$ is a purely inseparable extension of $Z$, in fact every element of $F$ satisfies an equation of the type $w^{p}-a=0, a$ in $Z$. By methods similar to the ones used in the case of characteristic zero we can show that $Z$ is of transcendence degree at least 2 over its prime field. Summing up we have

Theorem 3. Let $A$ be a Zorn ring properly containing its center $C$ and with all its zero divisors in $C$. Then $A$ is a division ring or the set of zero divisors coincides with the radical $R \neq(0)$ of $A$. In the latter case $A-R$ is a field $F$ and $C-R$ is a field $Z$. If $F$ has characteristic zero then it has transcendence degree at least 2 over $Z$. If $F$ has characteristic $p \neq 0$ then $F$ is a purely inseparable extension of $Z$ and $Z$ has transcendence degree at least 2 over its prime field.

CoROllaRY. Let $A$ be an algebraic alternative algebra over a quasialgebraically closed perfect field ${ }^{4}$ with all its zero divisors in the center, then $A$ is associative and commutative.

Theorem 3 generalizes a theorem of Herstein [2].

We now give examples of associative noncommutative Zorn rings with every zero divisor in the center. Let $H$ be the field of rational functions in two variables $x, y$ over the field of two elements. Let $J$ be the commutative algebra $H+H a$, with $a^{2}=0$. Finally let $A$ be the algebra $J \dot{+} J u \dot{+} J v \dot{+} J w$ over $J$ with the multiplication table:

4 A field is said to be quasi-algebraically closed and perfect if it satisfies the following: (i) There are no noncommutative alternative algebraic division algebras over it. (ii) If the field is of characteristic $p \neq 0$, it contains with every element its $p$ th root. Examples of such fields are given by algebraically closed fields and finite fields. 


\begin{tabular}{c|c|c|c} 
& $u$ & $v$ & $w$ \\
\hline$u$ & $x$ & $w$ & $x v$ \\
\hline$v$ & $a+w$ & $y$ & $y u+a v$ \\
\hline$w$ & $a u+x v$ & $y u$ & $x y+a w$
\end{tabular}

Then $A$ is an associative algebra of finite dimension over $H$ and thus is a Zorn ring. The zero divisors are all the multiples of $a$ and so lie in the center. However $(u, v) \neq 0$, and $A$ is noncommutative. Here $Z=H$ and $F=H\left(x^{1 / 2}, y^{1 / 2}\right)$.

Our second example is obtained as follows. Let $P$ be the field of all rational numbers and let $J$ be the commutative algebra $P+P a$, with $a^{2}=0$. Let $B$ be the algebra over $J$ with basis elements $u_{i j}, i, j=0,1,2, \cdots$, with the multiplication

$$
u_{i j} u_{k h}=u_{i+k, j+h}-j k a u_{i+k-1, j+h-1} \text {. }
$$

Then $B$ is an associative, noncommutative algebra with all its zero divisors in the center. It is, however, not a Zorn algebra, but we can embed $B$ in an algebra $A$ of right quotients using the method outlined on pp. 118-119 of [4]. It is only necessary to verify that for any two elements $\alpha$ and $\beta$ of $B$ with $\beta$ not a zero divisor, there exist elements $\alpha_{1}, \beta_{1}$ of $B$ such that $\beta \alpha_{1}=\alpha \beta_{1}$, with $\beta_{1}$ not a zero divisor. For $\beta_{1}$ we choose $\beta^{2}$ and set $\alpha_{1}=2 \alpha \beta-\beta \alpha$. To see that $\alpha_{1}$ and $\beta_{1}$ actually satisfy the condition it is only. necessary to note that all commutators of $B$ lie in the center. The algebra $A$ is then seen to be a Zorn algebra with all its zero divisors in the center. In this case $Z=P$ and $F=P(x, y), x=\bar{u}_{10}, y=\bar{u}_{01}$ transcendental over $P .{ }^{5}$

The method of the last example can be used to treat the case of an arbitrary associative ring $B$ with all its zero divisors in the center. In fact such a ring is either a domain of integrity or it has nonzero zero divisors. In the latter case if it properly contains its center it can be embedded in a right quotient ring $A$. Indeed, exactly the same $\alpha_{1}$ and $\beta_{1}$ as in the second example can be chosen. The ring $A$ is an associative ring properly containing its center $C$ and with all of its zero divisors in $C$. The zero divisors then form an ideal $I$ and again we have fields $F=A-I$ and $Z=C-I$. Since $A$ is noncommutative, the structure of $F$ over $Z$ must be exactly the same as in Theorem 3.

4. Nilpotent elements in the center. We now study alternative rings in which all the nilpotent elements lie in the center. However

S Added in proof March 9, 1953. It can easily be seen that $R$, the radical of $A$, is $a A$, so that $R^{2}=0$. However, the algebra $A$ provides a counter-example to the principal Wedderburn theorem: If $A=R+P(x, y)$, it would be commutative. 
we first of all restrict ourselves to the associative case. An easy computation shows that all the idempotents lie in the center $[3$, Lemma 2]. We first prove a result which is stated as an exercise in some lecture notes of Professor Kaplansky.

LEMMA 2. Let $A$ be an associative ring with the descending chain condition on right ideals. Suppose that all the nilpotent elements lie in the center of $A$. Then $A$ is a direct sum of a commutative nil ring and a finite number of rings each of which has all its zero divisors in the center.

Proof. Let $e_{1}, \cdots, e_{n}$ be a maximal set of orthogonal idempotents in $A$. If $e=\sum e_{i}, A(1-e)$ is nil and $A e_{i}$ is a two-sided ideal since $e_{i}$ is in the center. Thus $A=A(1-e) \oplus A e_{1} \oplus \cdots \oplus A e_{n}$. Since $e_{1}, \cdots, e_{n}$ is a maximal set of orthogonal idempotents, the only idempotent in $A e_{i}$ is $e_{i}$, its unit. Suppose $a$ is a zero divisor in $A e_{i}$; then $a b=0, b \neq 0$. If $a$ were non-nilpotent, since $A e_{i}$ is in particular a Zorn ring, there would be an $a^{\prime}$ in $A e_{i}$ such that $a^{\prime} a=e_{i}$. This would lead to $b=0$, a contradiction.

The only other class of rings we study in this connection are alternative algebraic algebras. We have first

Lemma 3. Let $A$ be an associative algebraic algebra over a quasialgebraically closed perfect field. Suppose that all the nilpotent elements of $A$ are in the center. Then $A$ is commutative.

Proof. Since the radical $R$ of $A$ is a nil ideal $R$ is commutative. Furthermore $R$ consists of all nilpotent elements of $A$ so that $A-R$ has no nonzero nilpotents. Then the corollary to Theorem 6 of [5] and the properties of the base field show that $A-R$ is also commutative. Thus $R$ and $A-R$ are locally finite, and so, by Theorem 15 of [5], $A$ is also locally finite. Hence if $x, y$ are any two elements of $A$ they generate a finite-dimensional algebra $D$, with all its nilpotents in the center. Lemma 2 in conjunction with the corollary to Theorem 3 shows that $D$, and hence $A$, is commutative.

To extend this result to an alternative algebraic algebra satisfying the conditions of Lemma 3 we proceed as follows: We consider the subalgebra generated by two elements of $A$. By the Theorem of Artin it is associative and so we can apply Lemma 3 to see that it, and hence $A$, is commutative. If the base field $\Phi$ is not of characteristic 3 it follows immediately that $A$ is also associative $[1,(2.6)]$. If however $\Phi$ is of characteristic 3 we first note that the commutativity of $A$ implies that $x \rightarrow(x, y, z)$ is a derivation. Thus $x^{3}$ is in the center of $A$ for all $x$ in $A$. Now we consider the finite-dimensional commutative associative algebra $\Phi(x)$ generated by $x$ over $\Phi$. If $R(x)$ is the radical of $\Phi(x), \Phi(x)-R(x)$ is a direct sum of finite algebraic extension fields 
of $\Phi$. Since $\Phi$ is perfect each of these is also, and so every element of $\Phi(x)-R(x)$ has a cube root. Thus $x=y^{3}+r$ where $y$ is in $A$ and $r$ is nilpotent, and this implies that $x$ is in the center of $A$. We have proved

THEOREM 4. Let $A$ be an alternative algebraic algebra over a quasialgebraically closed perfect field. Suppose that all the nilpotent elements lie in the center of $A$. Then $A$ is associative and commutative.

5. Banach algebras. We now give results analogous to those of $\$ \$ 2$ and 3 for Banach algebras. In what follows the Banach algebras will always be taken as real, i.e. the underlying Banach space is a vector space over the real numbers. The concept of a left zero divisor is replaced by that of a left topological zero divisor: $x$ is a left topological zero divisor if there exists a sequence $y_{n},\left\|y_{n}\right\|=1$, such that $x y_{n} \rightarrow 0$. We recall the definition of the spectrum, $s(x)$, of an element $x$ of a real Banach algebra. The complex number $\lambda=\alpha+i \beta \neq 0$ is in $s(x)$ if and only if $\left(x^{2}-2 \alpha x\right) / \alpha^{2}+\beta^{2}$ is not quasi-regular. For a discussion of $s(x)$ as well as the definition of the radical we refer to [6] and [11]. Here we only note that $s(x)$ is a bounded closed subset of the complex plane.

Theorem 5. Let $A$ be a real Banach algebra. Suppose that the set $L \neq A$ of left topological zero divisors forms a left ideal. Then $L=R$, the radical of $A$, and $A-R$ is the reals, complexes, or quaternions. Conversely, if $A-R$ is the reals, complexes, or quaternions, $L=R$ or $A$.

Proof. It is well known that $R$ is contained in $L$. Let $a$, not in $R$, be a left topological zero divisor. Then we may assume that $s(a) \neq 0$, for since $a$ is not in $R$ some left multiple of $a$ has that property. Let $\lambda_{0}=\alpha_{0}+i \beta_{0}$ be a nonzero boundary point of $s(a)$. Then, by Lemma 3.3 of [6], $1+\left(a^{2}-2 \alpha_{0} a\right) / \alpha_{0}^{2}+\beta_{0}^{2}$ and hence $\alpha_{0}^{2}+\beta_{0}^{2}-2 \alpha_{0} a+a^{2}$ is a left topological zero divisor. ${ }^{6}$ Now if $b$ is any element of $A,-2 \alpha_{0} b a$, $b a^{2}$, and $b\left[\alpha_{0}^{2}+\beta_{0}^{2}-2 \alpha_{0} a+a^{2}\right]$ are all elements of $L$. Since $L$ is a left ideal this implies that $\left(\alpha_{0}^{2}+\beta_{0}^{2}\right) b$, and hence $b$, is in $L$. This contradicts $L \neq A$, so $L=R$. Thus $b\left[1+\left(a^{2}-2 \alpha_{0} a\right) / \alpha_{0}^{2}+\beta_{0}^{2}\right]$ is in $R$ for all $b$ in $A$ and for $\lambda_{0}=\alpha_{0}+i \beta_{0}$ a nonzero boundary point of $s(a)$. Hence $A-R$ has a right unit $\bar{u}$. But if $u$ is a representative of $\bar{u}$ in $A$, $u\left[1+\left(b^{2}-2 \alpha^{\prime} b\right) / \alpha^{\prime 2}+\beta^{\prime 2}\right]$ is in $R$, where $b$ is not in $R$ and $\lambda^{\prime}=\alpha^{\prime}+i \beta^{\prime}$ is a nonzero boundary point of $s(b)$. Thus every nonzero element of $A-R$ has a right inverse, so that $A-R$ is a division algebra. But

\footnotetext{
- Even if there is no unit we may evidently speak of $\lambda+x$, where $\lambda$ is a scalar, as being a left topological zero divisor.
} 
the only real Banach division algebras are the reals, complexes, or quaternions.

If $A-R$ is the reals, complexes, or quaternions, since $s(a)=s(a+R)$, $s(a)$ consists of at most two points for all $a$ in $A$. Thus by Theorem 1.5 of [11] either $L=A$ or there exists a unit $e$ in $A$. In the latter case if $a$ is not in $R$ we know that there is an $a^{\prime}$ in $A$ such that $a^{\prime} a=e+r, r$ in $R$. But $e+r$ has an inverse in $A$, so that $a$ has an inverse also, thus $L=R$.

Corresponding to Theorem 3 we have

TheOREM 6. Let $A$ be a real Banach algebra with all its topological zero divisors in the center, $C$. Then $A$ is either the quaternions or it is commutative.

Proof. If $A$ has no nonzero topological zero divisors it is well known that $A$ is the reals, complexes, or quaternions. Suppose now that nonzero topological zero divisors are present and lie in $C$. If $A$ properly contains $C$ the same arguments as in $\$ 3$ show that the set of topological zero divisors is an ideal and so must coincide with $R$ the radical of $A$. Since just as before the commutators, $(x, y)$, lie in $R, A-R$ is the reals or complexes. However, $C$ is also a real Banach algebra with no topological zero divisors outside $R$. Combining Theorem 5 with that fact that $A$ contains $C$ properly we see that $C-R$ is the real field. Then $A-R$ is a quadratic extension of $C-R$. The same reasoning as in the proof of Theorem 3 then shows that $A$ must be commutative.

Kaplansky ${ }^{7}$ has shown that a complex $B^{*}$ algebra with all its nilpotents in the center is commutative. This theorem provides an analogue to our Theorem 4.

\section{BIBLIOGRAPHY}

1. R. H. Bruck and E. Kleinfeld, The structure of alternative division rings, Proceedings of the American Mathematical Society vol. 2 (1951) pp. 878-890.

2. I. N. Herstein, $A$ proof of a conjecture of Vandiver, Proceedings of the American Mathematical Society vol. 1 (1950) pp. 370-371.

3. - A generalization of a theorem of Jacobson, Amer. J. Math. vol. 73 (1951) pp. 756-762. 1943.

4. N. Jacobson, The theory of rings, Mathematical Surveys, vol. 2, New York,

5. - Structure theory for algebraic algebras of bounded degree, Ann. of Math. vol. 46 (1945) pp. 695-707.

6. I. Kaplansky, Normed algebras, Duke Math. J. vol. 16 (1949) pp. 399-418.

7. - Topological representation of algebras, Trans. Amer. Math. Soc. vol. 68 (1950) pp. 62-75.

\footnotetext{
7 To be published in the Report on Topological Algebra.
} 
8. - Semi-simple alternative rings, Portugaliae Mathematica vol. 10 (1951) pp. 37-50.

9. N. H. McCoy, Subdirectly irreducible commutative rings, Duke Math. J. vol. 12 (1945) pp. 381-387.

10. —, Rings and ideals, The Carus Mathematical Monographs, no. 8, Baltimore, 1948.

11. C. E. Rickart, The uniqueness of norm problem in Banach algebras, Ann. of Math. vol. 51 (1950) pp. 615-628.

12. M. F. Smiley, The radical of an alternative ring, Ann. of Math. vol. 49 (1948) pp. 702-709.

The Univers ity of Michigan 